\title{
Les acteurs noirs du cinéma français : de l’ombre à la lumière ?
}

\section{Aurélia Mouzet, Université d'Arizona}

Un tour d'horizon du cinéma français au vingtième siècle signale une présence spectrale de comédiens noirs à l'écran, présence qui tend d'ailleurs à décroître dès que l'on considère le nombre de premiers rôles qui leur ont été accordés. Lorsque l'on se penche sur l'influence des artistes noirs sur la scène française et au-delà se dégage un paradoxe : s'ils apparaissent dans le spectacle français dès le dix-huitième siècle (avec notamment le fameux Chevalier St George $^{1}$ ), ils demeurent pourtant des figures spectrales dans la mémoire collective. Seuls quelques noms restent en effet gravés dans l'histoire du spectacle français : le clown Chocolat (1878-1917), Habib Benglia (1895-1960), Feral Benga (1906-1957) et bien sûr Joséphine Baker (1906-1975). La prise de conscience de ce manque de reconnaissance accordé aux artistes noirs du spectacle français a, ces dernières années, engendré d'éminents travaux de recherche. L'on pense notamment aux ouvrages de Sylvie Chalaye, Gérard Noiriel, Nathalie Coutelet ou encore Régis Dubois qui ont su mettre en lumière le rôle important que ces artistes, trop souvent oubliés, ont joué dans l'évolution du spectacle en France.

La sortie en 2011 du film Intouchables d'Olivier Nakache et d'Éric Toledano, avec à l'affiche François Cluzet et Omar Sy, et le César reçu par Sy pour sa prestation semblaient pourtant annoncer la naissance d'une nouvelle ère : une vague multiculturelle qui aurait déferlé sur le cinéma français pour ouvrir enfin la voie à des comédiens noirs de talent jusque-là cantonnés aux rôles mineurs, par trop souvent stéréotypés. Avec ses dix-neuf millions d'entrées, Intouchables est désormais, comme l'écrit Charlie Michael, «largement reconnu comme le "phénomène culturel” français de l'année 2011 »(123). Dès sa sortie, le film a en effet connu un succès considérable. Dans un contexte de crise économique qui avait engendré avec elle une montée nationaliste ${ }^{2}$, le film apparaissait alors comme une promesse de réconciliation entre citoyens d'une France résolument «black-blanc-beur ». La réception du film fut toutefois foncièrement différente aux États-Unis. Comme le rappelle Michael, le principal détracteur d'Intouchables fut Jay Weissberg, critique proéminent de la revue Variety, qui perçut le film comme « overtly racist, pronouncing it guilty of flagrant "Uncle Tom-ism" » (133). Cette accusation, pour le moins radicale, a aussi entaché l'image de cette comédie dans l'hexagone, et d'autres, comme Régis Dubois, ont rejoint Weissberg dans sa critique acerbe du film. Les commentaires de Weissberg apparaissent toutefois particulièrement révélateurs de 
l'extrême difficulté à aborder les questions d'ethnicité aux États-Unis sans tomber dans le débat racial. Ainsi que le précise Pierre W. Orelus :

It seems as if people feel more comfortable or safer talking about race in private settings than doing so publicly, as public discussions revolved around race often make certain people uncomfortable, especially those who are racially privileged and/or are unwilling to challenge the White status quo, which has been oppressive to People of Color, particularly the poor ones. (575)

Bien que l'analyse d'Orelus soit, à maints égards, particulièrement pertinente, il est quelque peu regrettable qu'elle ne fasse aucun cas de l'une des difficultés fondamentales qui réside dans la prise en compte du discours racial pour les Américains blancs. Il n'est bien sûr pas question, ici, de nier l'existence du « white privilege » et la persistance, fort déplorable, de ce que l'on appelle la « ligne de couleur », mais plutôt de mettre ces réalités états-uniennes en perspective en rappelant que, du point de vue blanc, aborder la question de l'ethnicité est aussi, parfois, courir le risque d'être perçu comme raciste. Car si les artistes et intellectuels noirs entendent certes déconstruire une forme d'essentialisme identitaire au sein duquel l'histoire et les sociétés occidentales ont arbitrairement enfermé l'éthos noir, l'Américain blanc est, quant à lui, souvent perçu, par défaut, à l'aune d'un passé esclavagiste. Le choix des mots qu'il emploie est donc toujours à double tranchant. Ceci explique en partie pourquoi le concept de « color-blindness » est l'attitude — sans doute maladroitement - adoptée par certains pour pallier le risque d'incompréhension et éviter ainsi de telles accusations.

La critique de Weissberg signale par ailleurs très clairement cette fâcheuse tendance à préférer taire les inégalités plutôt que de les mettre au jour. Ce qui dérange dans Intouchables est avant tout le parallèle que le film établit entre les inégalités sociales et l'ethnicité ${ }^{3}$. Il véhicule ainsi, dans une certaine mesure, une image stéréotypée des communautés noires et pauvres de France, sans emploi et «zonant» dans les banlieues. Si l'on ne peut certes que regretter la caractérisation quelque peu clichée du personnage de Driss, l'on ne peut toutefois nier une certaine réalité : les banlieues françaises sont effectivement des espaces multiculturels où cohabitent, dans des conditions plus ou moins salubres, des communautés issues, ou non, de l'immigration ayant, entre autres, pour point commun leurs revenus modérés. Il convient à ce titre de souligner qu'au contraire de ce que l'opinion publique voudrait faire croire, si la violence et la pauvreté sont certes des réalités sociales au sein des banlieues de France, elles sont surtout le fait de choix politiques peu judicieux et ne sont en rien l'illustration de tares quelconques qui caractériseraient les individus eux-mêmes ${ }^{4}$. Le succès phénoménal du film n'en demeure pas moins en demi-teinte. En effet, si nombreux sont ceux qui se sont réjouis de la césarisation de $\mathrm{Sy}$, un tel succès a aussi permis de mettre au jour des inégalités flagrantes au 
sein d'une République qui se voulait — et se veut toujours — résolument multiculturelle. Comment expliquer en effet la présence si disparate de comédiens noirs dans le cinéma français ? Pourquoi semblent-ils condamnés à jouer des rôles stéréotypés trop souvent nourris de clichés hérités de l'imaginaire colonial ? C'est à ces multiples interrogations que cet article tentera de répondre en cherchant à mettre au jour l'évolution des personnages noirs dans le paysage cinématographique français. À travers l'analyse de trois auvres : Zouzou (Marc Allégret), La Noire de (Ousmane Sembène) et Intouchables (Toledano et Nakache), nous verrons dans quelle mesure les rôles offerts aux comédiens noirs contribuent à enfermer les individus dans le carcan d'une identité stéréotypée imposée par un monde blanc qui peine encore à se défaire des stigmates d'un passé colonial toujours présent. Nous modérerons toutefois cette dimension en soulignant l'impérieuse nécessité de parler de sujets brûlants et ce, même et surtout au cinéma et dans l'art, car bien que leur traitement thématique court le risque de nourrir les stéréotypes, ne pas en faire cas reviendrait à faire du «politiquement correct » et contribuerait ainsi uniquement à attiser les tensions intercommunautaires en France. C'est effectivement dans le dialogue et la mise au jour d'inégalités persistantes que réside une meilleure compréhension des conflits qui agitent la France aujourd'hui.

\section{De Jezebel à Oncle Tom : le carcan identitaire de l'éthos noir sur les écrans français}

Le personnage incarné par Joséphine Baker dans Zouzou est emblématique des rôles stéréotypés imposés aux Noirs dans le but de satisfaire la soif d'exotisme d'une audience blanche dont l'imaginaire collectif regorge de clichés véhiculés par les récits de voyage, les médias et la littérature, tous fortement inspirés par les théories coloniales. Le corps de Zouzou est ainsi érotisé de manière paroxystique comme en témoigne la danse lascive qu'elle effectue à l'insu du directeur du théâtre et qui témoigne du stéréotype d'hypersexualité associé à la femme noire. La chanson que la jeune femme interprète en meneuse de revue signale par ailleurs la fâcheuse tendance de la société blanche à considérer tous les Noirs comme une seule et même entité. En effet, bien que Baker soit américaine, Zouzou est prétendument née dans une île de l'archipel polynésien. Elle nous est ensuite présentée comme venant de Martinique pour finalement chanter : «Qui me rendra mon pays, Haïti ». Le caractère diffracté de l'identité noire, mis ici en lumière par les multiples visages imposés à Zouzou, signale la difficulté pour une société qui fut nourrie par les clichés coloniaux à considérer l'individu noir dans sa subjectivité : d'où qu'il vienne, le Noir court le risque de devenir l'objet de fantasmes généralisant qui tendent à nier son individualité. Dans son essai «The Negro Art Hokum », George Schuyler, figure proéminente du mouvement de la Harlem Renaissance, s’est insurgé 
contre le recours aux stéréotypes et ce, même dans le but de renouveler une identité «nègre » bafouée par des siècles d'oppression :

Aside from his color, which ranges from very dark brown to pink, your American Negro is just plain American. Negroes and whites from the same localities in this country talk, think, and act about the same. Because a few writers with a paucity of themes have seized upon imbecilities of the Negro rustics and clowns and palmed them off as authentic and characteristic Afroamerican behavior, the common notion that the black American is so «different» from his white neighbor has gained wide currency. The mere mention of the word «Negro » conjures up in the average white American's mind a composite stereotype of Bert Williams, Aunt Jemima, Uncle Tom, Jack Johnson, Florian Slappey, and the various monstrosities scrawled by the cartoonists. Your average Afroamerican no more resembles this stereotype than the average American resembles a composite of Andy Gump, Jim Jeffries, and a cartoon by Rube Goldberg. (97)

Loin d'offrir un cadre épistémologique à même de saisir la réalité de l'altérité en termes scientifiques, le recours à l'essentialisme procède d'un mouvement unilatéral qui analyse l'Autre en tant qu'objet, fixé à la fois dans l'espace et le temps, lui refusant ainsi la fonction de sujet et le devenir. Ce regard porté sur l'autre se nourrit de fantasmes et de pulsions réfrénées de telle sorte que pseudo-réalité et imaginaire finissent par cristalliser les représentations de l'altérité et nient alors l'identité individuelle en la noyant dans son rapport au collectif.

Au sein des discours panafricanistes, l'essentialisme, revendiqué dans le but de fonder une conscience noire transnationale, fut toutefois la meilleure arme pour contrer la négation même de leur identité par les Blancs. Pour les Noirs américains, le début du vingtième siècle était aussi le creuset d'injustices encore plus arbitraires et inexplicables que par le passé dans la mesure où elles ne se « justifiaient » plus par l'esclavage. Les lois Jim Crow et la ségrégation réfutaient, par leur existence même, la possibilité pour les Afro-Américains de jouir d'une liberté et d'une égalité absolues. Puiser dans les racines africaines de tout un peuple la force de résister était pour eux le moyen de rallier une communauté brisée autour d'une cause commune, tout en lui permettant de recouvrer la dignité dont la haine raciale l'avait jusqu'alors privée. A l'instar des États-Unis, la France a, elle aussi, véhiculé de nombreux stéréotypes sur les Noirs. Ces clichés furent réappropriés et repris à des fins de propagande par le mouvement de la Négritude afin de contrer la déshumanisation des communautés noires et la domination culturelle imposée par les puissances coloniales.

Même s'il est indéniable que Joséphine Baker ait incarné des rôles qui l'enfermaient dans une certaine catégorie d'altérité exoticisée pour les besoins du public, elle n'en a pas moins donné aux spectateurs ce qu'ils attendaient afin de s'en servir à son avantage. Elle a 
ainsi utilisé cette image pour faire carrière et trouver une alternative à l'avenir peu radieux que lui promettait l'Amérique ségréguée qui l'avait vue naître. Tout comme Zora Neale Hurston a su utiliser le folklore afro-américain et sa maîtrise des dialectes afin d'en nourrir ses œuvres pour qu'elles répondent aux attentes du lectorat blanc, Joséphine Baker a, quant à elle, exploité son talent pour la danse et sa sensualité pour créer un personnage populaire dont le public blanc était fou. Elle a su détourner les codes à son profit et bien que Zouzou ou Princesse Tam-Tam ne soient pas considérés comme des chefs-d'œuvre cinématographiques, ils apparaissent particulièrement révélateurs de cette difficulté de choisir sa propre identité lorsqu'on est noir, dans la mesure où celle-ci est souvent dérobée, déformée voire imposée par la culture dominante. En se réappropriant les stéréotypes projetés par les Blancs sur son identité de femme noire, Baker s'est paradoxalement reconstitué une identité singulière. Dans une certaine mesure libérée du carcan colonial, elle lui a permis de mener son combat contre l'exclusion :

She was courageous, too, in her opposition to racism in America, always refusing to appear before segregated audiences and campaigning for the Civil Rights movement. Her final, more utopian project was the « rainbow tribe » of a dozen orphan children from different parts of the world whom she adopted and brought up as an example of racial harmony in her chateau in the Dordogne. (Robin Buss 36)

Le choix fait par Baker d'assumer complètement cette sensualité exacerbée fait écho à la réappropriation du terme «Nigger» au sein, notamment, de la culture hip-hop africaine américaine. Plutôt que de se laisser enfermer au sein d'une identité exclusivement négative, véhiculée par des stéréotypes ou des termes dégradants, la jeunesse noire américaine choisit de se réapproprier ce qui se trouve au fondement même des critiques les plus acerbes afin d'en faire un élément identitaire positif. Cette réappropriation du soi par soi est, malgré ce qu'en disent de nombreux critiques, aussi sensible dans Intouchables. Comme nous le disions plus haut, Dubois rejoint Weissberg dans son attaque du film qu'il qualifie, lui aussi, de raciste :

Le problème finalement ce n'est pas tant qu'il existe des personnages comme Driss au cinéma. Je n'ai aucun problème avec les stéréotypes, il en faut, surtout dans la comédie. Non, le problème c'est qu'il n'y a que des personnages comme Driss dans le cinéma français. [...] Pourquoi ne pourrait-il donc pas y avoir des rôles de Noirs «normaux », ni voyous ni comiques, façon père de famille tranquille, avec un emploi «normal »? Pourquoi pas des personnages de Noirs journalistes ou militants politiques, ou même chefs d'entreprises, artistes peintres ou encore écrivains ? Et pourquoi pas un rôle de président noir comme aux USA - à l'image de Deep Impact (1998) ou de la série 24 heures chrono (2001) ? Sans doute cela arrivera-t-il un jour. Ne vient-on pas d'apprendre que Joey Starr est nommé aux Césars pour son rôle à contre-emploi de flic émouvant dans Polisse ? Espérons juste qu'il ne faudra pas attendre 30 ans... (par. 8) 
Or, si l'on ne peut nier que le personnage de Driss soit le stéréotype du Noir de cité qui fume du shit, vole et pointe à l'ANPE tout en étant à la fois nounou et comique, il n'en échappe pas moins à la caractérisation binaire dans la mesure où il fait aussi montre de courage, de perspicacité et d'une extraordinaire faculté d'adaptation qui lui permettent de venir à bout des barrières que ses racines populaires auraient pu mettre sur sa route. Si nous rejoignons Dubois lorsqu'il regrette que l'on ne compte plus les rôles de voyous des cités, d'agents de sécurité ou encore de bonnes à tout faire incarnés par des comédiens noirs, refuser le droit à la représentation aux jeunes noirs de banlieues, sous prétexte qu'ils incarnent un stéréotype, tend malheureusement une fois de plus à confiner les marges aux marges. Que reproche-t-on réellement aux stéréotypes? Ceux qui procèdent de l'imaginaire colonial véhiculent une image dévalorisante de l'altérité qui a parfois été intériorisée par les individus, engendrant chez certains une fragmentation identitaire menaçant à la fois l'équilibre de la psyché, mais aussi le potentiel de réussite des individus. S'il est certes regrettable que les comédiens noirs soient enfermés dans ce type de rôles, est-il plus judicieux de refuser le droit à la représentation de tous ces jeunes de banlieue sous prétexte qu'ils incarnent un stéréotype? La violence, l'exclusion, le racisme ne sont malheureusement pas que des chimères dans les banlieues françaises et sans doute la critique profiterait-elle d'une relation plus concrète au monde plutôt que de se cantonner à théoriser un monde perçu à travers le prisme d'une pensée bourgeoise dont elle peine à se détacher. Bien sûr, les Noirs ne sont pas tous videurs, voyous et marginalisés et il est effectivement indispensable que l'art et la fiction s'ouvrent à la mixité sociale en diversifiant la manière dont ils y sont représentés. Il est néanmoins tout aussi important de continuer à parler de ces jeunes et des problématiques qu'ils rencontrent dans les cités de France. Il ne s'agit pas bien sûr de les enfermer au sein de représentations identitaires exclusivement négatives, mais plutôt de mettre en lumière la vie qui continue d'affleurer dans ces quartiers qui dérangent la France bien-pensante. Il est tout un pan de la culture des banlieues - la musique, la famille, l'amitié - qui a été mis en lumière dans le film et qui a été balayé et nié par la critique. Weissberg, rejoint par le très français journal Libération, dénonce mièvrerie, sadisme et racisme :

[L]e personnage de Driss est «traité comme un singe de compagnie (avec toutes les insinuations racistes que comporte ce mot), qui apprend au blanc coincé à s'amuser, en remplaçant Vivaldi par Boogie Wonderland, et en lui montrant comment on bouge sur la piste de danse. C'est pénible de voir Sy, un acteur joyeusement charismatique, dans un rôle qui se détache à peine de l'époque de l'esclavage, dans lequel il divertit le maître blanc, en endossant tous les stéréotypes raciaux et de classe. (par. 3) 
Tandis que le New York Times « reproche à Intouchables de 'reposer largement sur la nature effrontée de Driss, qui joue le rôle stéréotypé du loser inculte de banlieue » et Weissberg d'enfoncer le clou en affirmant «Intouchables pourrait séduire un public qui ne réfléchit pas trop, le tout dans une atmosphère détestable» (Libération, par. 6, 4). Le problème fondamental réside finalement moins dans le film lui-même que dans le prosélytisme dont fait malheureusement preuve tout un pan de la critique. Cette dernière, persuadée de posséder le savoir absolu, se gargarise intellectuellement en discréditant la capacité d'analyse du grand public.

Le problème principal de l'analyse proposée par Weissberg de la scène Boogie Wonderland est son incapacité à s'extraire d'une réception bourgeoise des images qui lui sont renvoyées. La prise de vue est fort probablement à la source de cette analyse tronquée, dans la mesure où elle est transmise au spectateur au moyen de la focalisation interne. Un plan rapproché de Philippe qui regarde vers la piste de danse, l'on voit ensuite Driss danser et l'on entend Philippe appeler son nom en voix off. Les plans suivants alternent ensuite entre la piste de danse et Philippe qui, au contraire de ce qu'en dit Weissberg, ne regarde pas Driss avec simple amusement. Le public ressent en effet aussi toute la douleur éprouvée par cet homme à l'idée de ne jamais plus pouvoir se lever pour aller lui-même sur la piste de danse. Le problème de la focalisation interne, c'est qu'elle impose un filtre blanc sur l'image du Noir et c'est sans doute cela qui prête à confusion, car il est parfois aussi difficile au Blanc de se dégager de l'image de colon raciste, qu'il l'est au Noir de se défaire des stéréotypes imposés par la société blanche.

Ceux qui voudront voir du racisme dans Intouchables trouveront certainement de nombreux arguments pour nourrir leurs critiques, mais il importe de souligner que la relation de Philippe et Driss n'est pas fondée, comme certains voudraient le croire, sur des rapports de force hérités de la colonisation. Philippe n'est d'ailleurs pas moins stéréotypé que Driss dans le film : c'est un bourgeois qui apprécie l'opéra, Vivaldi et les tableaux de maîtres. Si Driss vient de banlieue, il n'en témoigne pas moins d'une certaine supériorité à l'égard de cette société policée, étouffée par les normes et les codes qu'elle s'impose. Il est libre, dans la mesure où il sait transgresser les règles austères de bienséance et goûte ainsi au plaisir de la « vraie » vie. L'humour dont il fait preuve lors de son entretien d'embauche, après avoir quitté Philippe, permet au personnage de dépasser le seul stéréotype. Si le comique ne demande pas de maîtrise intellectuelle, l'humour l'exige et l'aptitude de Driss à faire rire ne fait de ce fait en aucun cas de lui un bouffon, mais souligne plutôt sa perspicacité. Loin de n'être que le voyou de banlieue que certains ont vu dans son personnage, Driss témoigne à la fois d'une extraordinaire humanité 
et d'une faculté de résistance que seuls ceux qui se trouvent confrontés à l'adversité au quotidien savent développer. Il est en cela l'incarnation positive du jeune de cité qui réussit à s'en sortir avec les moyens qui lui sont donnés. Le fait que la fin reste ouverte et laisse le futur de Driss dans le flou offre au spectateur le choix d'y voir un avenir radieux ou non. Qu'il ne retourne pas dans sa cité est finalement assez révélateur, dans la mesure où ceux qui l'ont expérimentée savent que même si l'on n'oublie jamais d'où l'on vient, et si la cité nous fournit certes les armes pour lutter contre l'adversité, l'avenir est aussi ailleurs.

$\mathrm{Au}$ contraire de ce qu'en disent les critiques, Driss déconstruit finalement tous les stéréotypes car il apparaît comme l'incarnation de vraies valeurs dans un monde pourri par l'argent. Il est aussi cette promesse d'avenir que bon nombre refusent aux enfants des cités. Enfin, en tant qu'acteur noir, Sy est peut-être aussi, espérons-le, une promesse de renouveau. Comme le souligne Elikia M'Bokolo dans l'édito de l'émission de RFI consacré à l'ouvrage de Nathalie Coutelet :

Ils sont assez nombreux aujourd'hui, ces artistes noirs venus d'Afrique, de la Caraïbe ou des Amériques. Certains jouissent même d'une grande visibilité. C'est une étape plutôt heureuse dans un long cheminement qui commence à la fin du [dix-neuvième] siècle. Un vrai parcours du combattant, dans une France paradoxale, républicaine, égalitaire et fraternelle, mais aussi coloniale et sourde à l'altérité. Comment Chocolat (1878-1917), Habib Benglia (1895-1960), Feral Benga (1906-1957) et même la plus célèbre, Joséphine Baker (1906-1975) se sont-ils insérés dans cet univers artistique et social à la fois chaleureux et impitoyable? (par. 1)

\section{Regards d'Afrique, images de France: une perspective transatlantique pour déconstruire l'imaginaire colonial}

Bien que le film de Sembène soit bien sûr sénégalais, le réalisateur a toutefois collaboré avec une équipe de production française. L'intégration de cette œuvre dans le corpus nous paraît, à ce titre, particulièrement riche de sens pour le sujet qui nous intéresse. Il s'agit en effet du premier long métrage réalisé par un cinéaste d'Afrique noire en collaboration avec une équipe française. Il réduit ainsi l'imposition du filtre blanc sur l'image du Noir et permet d'aborder la notion de stéréotype de manière transversale. Rachael Langford rappelle à cet égard que certaines lois visaient, à l'époque, à limiter la liberté d'expression des cinéastes africains, de peur que ceux-ci ne soient trop critiques à l'égard des anciennes puissances coloniales :

Until the mid-1960s, only non-Africans had depicted Africa on film. Indeed, such laws as the décret Laval of 1934, applying to French colonial possessions, had aimed at preventing Africans from making potentially critical films about their lives and their countries [...]. From the mid-1960s onwards, however, 
francophone Africans in particular turned the camera on themselves and their continent, for themselves and their continent, with increasing frequency and confidence. (13)

Le film de Sembène figure donc le renversement de point focal : si le personnage de Diouana n'est certes pas dénué de stéréotypes, ceux-ci sont traités par l'intermédiaire de la focalisation interne, ce qui permet dès lors au cinéaste de mener une réflexion à la fois politique et esthétique qui vise à leur déconstruction. Mbissine Thérèse Diop y tient le premier rôle, Diouana, une jeune femme sénégalaise qui travaillait comme nounou pour une famille française à Dakar et accepte de suivre sa patronne blanche lorsque celle-ci quitte le Sénégal. Dès le début du film, le silence de Diouana contraste avec la volubilité de ses patrons blancs. Aux multiples questions de ces derniers, elle ne répond que quelques éparses « Vi, Monsieur» ou « Vi, Madame. » Son mutisme donne lieu à des interprétations paradoxales. Il semble, dans un premier temps, faire écho à la représentation du stéréotype colonial du «bon sauvage » à la fois docile et silencieux. Toutefois, comme le précise Langston, le silence de Diouana est aussi et surtout une forme de résistance qu'elle oppose à l'oppression néocoloniale de ses patrons blancs. Résistance que le spectateur ne découvre qu'après plusieurs minutes en entendant la voix off exprimer les sentiments intérieurs de la jeune femme :

Although Malkmus and Armes argue [...] that the use of voice-over in La Noire de... colludes in the colonial silencing of the African by depicting a passive Diouana unable to speak on her own behalf, I would argue that it forms a vital challenge to such silencing. Firstly, it bears witness to the film's contestatory reversal of the narrative paradigms legitimating colonialism, because it allows a neo-colonized African 'I' to frame a travelogue-memoir. It thus allows the audience privileged access to Diouana 'for intérieur', her innermost being, while highlighting the extent to which her French employers tacitly deny that she possesses such interior.

And secondly, it foregrounds the extent to which Diouana chooses to present herself to the white world as solely as an indelible and unadministrable 'foreign body', as a strategy of resistance. (15)

Madame dépossède Diouana de sa féminité et lui impose le port d'un tablier pour lui rappeler que c'est elle qui exerce l'autorité : «Diouana, tu n'es pas à la noce. Ça fait trois semaines que tu es habillée comme ça. Enfin, change de tenue ! Privée de liberté dans sa propre chair, la jeune femme est totalement réifiée par les Blancs qu'elle côtoie en France comme le signale la remarque de l'homme qui l'embrasse : «Vous permettez Mademoiselle, je n'ai jamais embrassé de négresse ». N'étant plus à même de disposer de son corps comme elle l'entend, c'est en se réappropriant symboliquement ce corps dont on tente de la déposséder que Diouana développe ses stratégies de résistance. Peu avant de mettre fin à ses jours, elle réaffirme son refus absolu de se soumettre à ses patrons : «Plus jamais Madame me dira 
quelque chose. Jamais plus Madame me dira : "Diouana, fais du café !" Jamais plus : "Diouana, prépare-nous du riz !’ Jamais plus : "Diouana, enlève tes chaussures !” Jamais plus : “Diouana, lave la chemise de Monsieur !’ Jamais plus : “Diouana, tu es une fainéante !” Jamais je ne serai esclave !» La reprise anaphorique de la négation «jamais plus » souligne l'ampleur de la révolte de Diouana. Cette jeune femme que les Blancs ont voulu cantonner au rôle de bonne à tout faire en lui refusant tout divertissement, recouvre à la fin de sa vie la faculté de dire « je », et avec elle, le droit absolu de choisir elle-même son propre destin. Mais bien qu'elle atteigne son paroxysme avec la mort, la rébellion de Diouana s'était déjà manifestée peu après son arrivée. Selon Langston,

Her initial resistance to the object-identity in which she is cast takes the form of small refusals to regulate her body according to the patterns of work and rest that her employers require. She fails to get up at the appointed hour in the morning, thus forcing her mistress to prepare the family's breakfast herself; she spends much longer in the bathroom than she is allowed to. (19)

La signification symbolique du masque africain apparaît à ce titre particulièrement révélatrice des stratégies de résistance développées par les communautés noires pour contrer et survivre à l'oppression blanche. Le masque incarne, aux yeux des colons, le mystère, la sauvagerie et la tribalité qu'ils attribuent aux Africains. En l'épinglant sur ce grand mur blanc, les patrons de Diouana soulignent leur ignorance des coutumes africaines mais aussi leur incapacité à considérer l'autre en tant qu'égal. Langston écrit à cet égard : "[the mask's] location speaks as a misrecognition of its identity : the mask is perceived by the white family only as an art object, and their entire behaviour towards Diouana shows their (wilful ?) ignorance of the mask's standing as a gift implying reciprocity and equality of relations » (17). Le cadeau trône ainsi dans le salon, tout comme ils exhibent leur bonne africaine devant leurs amis. Diouana reprend ce masque lorsqu'elle comprend que la relation qu'elle entretient avec ses patrons, est fondée sur des valeurs qui n'en sont pas. La crise que pique Madame quand elle réalise que le masque lui a été repris, figure le déséquilibre qui fonde les rapports entre Blancs et Noirs à l'aune du régime colonial. Les premiers exigent bien souvent sans rien donner en retour. Le refus de la mère de Diouana d'accepter l'argent du patron après la mort de sa fille symbolise son rejet absolu de ce mode d'échanges biaisés, et illustre ainsi sa révolte à l'égard d'une société qui ne cesse de réifier les individus afin qu'ils servent la collectivité.

En se suicidant, Diouana s'est réapproprié de manière paroxystique le corps que les Blancs avaient voulu contraindre à la soumission. A travers le personnage de Diouana, Sembène érige le corps de la femme africaine en site de résistance particulièrement efficace, dans la mesure où il se sert des stéréotypes pour déconstruire le discours dominant. 
L'hypersexualité de la femme africaine est déjouée ici par le motif de la mort et, en un clin d'œil sarcastique à ces maîtres blancs, Diouana grave ainsi à jamais, dans l'esprit de ses patrons blancs, son image de Vénus noire qu'ils n'auront pas réussi à soumettre.

Le masque porté par le petit frère de Diouana lorsqu'il suit le patron blanc s'érige, par ailleurs, en symbole du poids de la culpabilité qui continuera de hanter les maîtres de Diouana où qu'ils aillent. Lorsque le petit garçon finit par retirer son masque, il devient alors une métaphore du continent africain et de tous les peuples africains auxquels la colonisation a imposé les masques de l'infériorité et de la sauvagerie, mais qui, malgré toutes les tentatives de clôture de l'identité sous le prisme colonial, conservent une identité propre et singulière qu'ils choisissent seuls de déterminer.

En conclusion, s'il est regrettable que la France peine à se défaire de cette conception «petit bourgeois » d'un monde au sein duquel les Noirs ne peuvent être représentés qu'au moyen de stéréotypes pour la plupart dégradants, le talent des comédiens et cette conscience accrue de la nécessité d'un changement leur a permis de déjouer, et ce, même à travers les rôles clichés qui leur ont été attribués, la puissance des stéréotypes en offrant une richesse de jeu qui emmène les personnages les plus caricaturaux vers l'humanité dans toute sa diversité. Personne n'aurait cru, il y a cinquante ans, que les États-Unis auraient eu, un jour, un président noir, aussi est-il possible d'espérer que Baker, Benga, Sy et les autres ouvriront la voie à un cinéma multiculturel à l'image du pays dont il est le produit. L'image aseptisée du petit bourgeois franco-français n'est plus en rien représentative de la France d'aujourd'hui. Si l'histoire n'a longtemps été que l'histoire des rois et des puissants, celle-ci s'intéresse désormais aux minorités. Il en est de même pour la littérature et la musique. Il est désormais grand temps que le septième art suive le mouvement et permette aux comédiens noirs et à tous ceux issus des minorités de briller aussi sur les écrans français.

\section{Bibliographie}

Allégret, Marc. Zouzou. Ciné-Aris productions et les Films H. Roussillon, 1934.

Anonyme. «Intouchables : un film qui flirte avec le racisme selon le site américain 'Variety' ». Libération. 8 décembre 2011. http://next.liberation.fr/cinema/2011/12/08/intouchables-un-film-qui-flirte-avec-leracisme-selon-le-site-americain-variety_780467. Consulté le 5 avril 2014.

Buss, Robin. «Josephine Baker an AMERICAN in PARIS ». Sight \& Sound 17. 3 (2007) : 36-38. 
Dubois, Régis. «Autour du film Intouchables : présence et absence des Noirs dans le cinéma français ». Danactu-résistance (2011). http://danactu-resistance.over-blog.com/articleautour-du-film-intouchables-presence-et-absence-des-noirs-dans-le-cinema-fran-ais94535554.html. Consulté le 3 mai 2014.

Langford, Rachael. «Black and White in Black and White : Identity and Cinematography in Ousmane Sembne's La Noire de.../Black Girl (1966)». Studies in French Cinema 1.1 (2001) : 13-21.

M'Bokolo, Elikia. « Artistes noirs du spectacle français ». Mémoire d'un continent. RFI 9 mars 2013.<http://www.rfi.fr/emission/20130914-artistes-noirs-spectacle-francaisrediffusion/>. Consulté le 9 mai 2014.

Michael, Charlie. «Interpreting Intouchables : Competing Transnationalisms in Contemporary French Cinema ». SubStance 43.1 (2014) : 123-137.

Nakache, Olivier et Eric Toledano. Intouchables. Gaumont et TF1 Films Production, 2011.

Orelus, Pierre W. «Unpacking the Race Talk». Journal of Black Studies 44.6 (2013) : 572589.

Schuyler, George Samuel. «The Negro Art Hokum ». Nation Associates (1926) : 96-99.

Sembène, Ousmane. La noire de. Filmi Domirev et Les Actualités françaises, 1966.

\footnotetext{
NOTES

${ }^{1}$ Escrimeur et musicien, métis né en Guadeloupe d'un père colon blanc et aristocrate et d'une mère esclave d'origine africaine (1745-1799).

${ }^{2}$ Montée nationaliste portée à l'attention du public lors de son paroxysme un an plus tard avec les $17,9 \%$ de votes remportés par le FN lors de l'élection présidentielle de 2012.

${ }^{3}$ Que nous refusons d'appeler « race » dans la mesure où nous considérons le concept même de race noire comme erroné et n'ayant aucune validité scientifique.

${ }^{4}$ Les coupes budgétaires décidées par le gouvernement Sarkozy en 2008 en matière d'éducation ont limité le champ d'intervention du dispositif RASED (Réseaux d'aides spécialisées aux élèves en difficulté) qui permettait notamment aux enfants de maternelle en difficulté de bénéficier d'un accompagnement personnalisé durant l'heure de déjeuner afin qu'ils acquièrent de meilleurs outils d'apprentissage. En supprimant de nombreux postes dans les établissements classés ZEP (Zone d'éducation prioritaire), le gouvernement a fait accentuer l'inégalité des chances, promettant ainsi à de trop nombreux jeunes de banlieues un avenir en point d'interrogation, faute de fournir aux écoles les moyens de combattre l'échec scolaire. Déscolarisés, parfois dès l'âge de douze ans, ces jeunes sont, à leur corps défendant, confrontés au chômage, à la pauvreté et à l'exclusion.
} 